\title{
Dynamics and heat and mass transfer under spreading of liquid-droplet aviation fuel in the atmosphere
}

\author{
Vladimir Arkhipov ${ }^{1}$, Irina Zharova ${ }^{1, a}$, Eugene Kozlov $^{1}$, and Aleksey Tkachenko ${ }^{2}$ \\ ${ }^{1}$ Tomsk State University, Scientific Research Institute of Applied Mathematics and Mechanics, \\ Tomsk, Russia \\ ${ }^{2}$ Tomsk State Pedagogical University, Tomsk, Russia
}

\begin{abstract}
A physical-mathematical model of dynamics and heat and mass transfer during spreading of liquid-droplet aviation fuel in the atmosphere is presented. The optimal emergency discharge height of kerosene for different Russian regions was evaluated based on the proposed mathematical model. The developed model can be used to select the height limit of reset kerosene, guaranteeing complete evaporation of the droplets in the atmosphere to the different aircraft velocities.
\end{abstract}

\section{Introduction}

There is a need of numerical prediction of consequences of emergency situations arising during the air transportation of cargo and passengers in connection with the active using by aviation means of airspace. From the fuel tank forcibly ejected kerosene come into an atmosphere and dissipated there. In this, part of the fuel evaporates, contaminating the atmosphere, and the non-evaporated droplets kerosene fall to the ground surface. Herewith the some part of fuel is evaporating, contaminating the atmosphere, and the non-evaporated kerosene droplets fall to the ground surface.

In this paper, a mathematical model [1] used to assess the extent of environmental pollution and to select the optimum height of accidental discharge of aviation fuel. Motion of the liquid-droplet cloud of aviation fuel in the atmosphere was examined with account to evaporation and possible splitting of liquid particles. It was estimated the emergency discharge height required for complete evaporation of drops of kerosene in the regions that are significantly different according to seasonally-climatic conditions. Calculations were performed for different months with the corresponding known time-averaged of distributions atmosphere parameters [2].

\section{Modeling the evolution of droplets cloud of aviation fuel in the atmosphere}

The physical model is based on the following assumptions [1,3]. It was assumed that the force of gravity, wind load and the drag force act on every drop of aerosol cloud. The heat transfer between the droplet

\footnotetext{
${ }^{a}$ Corresponding author: zharova@niipmm.tsu.ru
}

This is an Open Access article distributed under the terms of the Creative Commons Attribution License 4.0, which permits unrestricted use, distribution, and reproduction in any medium, provided the original work is properly cited. 
and the surrounding air and evaporation of the droplet were taken into account. Under the assumptions made, the process under study is described by the following system of equations

$$
\left\{\begin{array}{l}
\frac{d u_{s}}{d t}=\phi\left(u-u_{s}\right), \frac{d v_{s}}{d t}=\phi\left(v-v_{s}\right), \frac{d w_{s}}{d t}=\phi\left(w-w_{s}\right)+g, \\
\frac{d T_{s}}{d t}=\frac{3}{2} \frac{\lambda}{r_{s}^{2} \rho_{s} c_{p}} \mathrm{Nu}\left(T-T_{s}\right)-\frac{q q_{v a p} m_{v a p}}{m_{s} c_{p}}, \\
\frac{d r_{s}}{d t}=-\frac{G_{s}}{4 \pi \rho_{s} r_{s}^{2}}, \\
\frac{d x}{d t}=u_{s}, \frac{d y}{d t}=v_{s}, \frac{d z}{d t}=w_{s},
\end{array}\right.
$$

where $u_{s}, v_{s}, w_{s}$ are the components of the droplet velocity $\vec{U}_{s} ; u, v, w$ are the components of the wind velocity vector $\vec{U} ; \phi=\frac{3 \rho}{8 \rho_{s} r_{s}} C_{D}\left|\vec{U}-\vec{U}_{s}\right| ; \rho$ is the air density; $\rho_{s}$ is the liquid density; $C_{D}$ is the aerodynamic drag coefficient; $r_{s}$ is the droplet radius; $g$ is the acceleration of free fall; $T$ is the absolute air temperature; $T_{s}$ is the temperature of a droplet (the averaged over the volume); $\lambda$ is the coefficient of the gas thermal conductivity; $c_{p}$ is the specific heat capacity of liquid; $\mathrm{Nu}=f(\mathrm{Re}, \mathrm{Pr})$ is the Nusselt number; $\operatorname{Re}=2 \rho\left|\vec{U}-\vec{U}_{s}\right| r_{s} / \mu$ is the Reynolds number of the relative movement; Pr is the Prandtl number; $q_{\text {vap }}$ is the specific heat of the liquid evaporation; $m_{\text {vap }}$ is the mass of evaporated liquid; $m_{s}$ is the droplet mass; $G_{s}$ is the liquid mass evaporating from the droplet surface per unit time (evaporation rate).

Assuming a spherical drop shape the values of the number $\mathrm{Nu}$ and aerodynamic drag coefficient $C_{D}$ were determined depending on the flow regime $[4,5]$ :

- Stokes flow, $\mathrm{Re} \leq 1: \mathrm{Nu}=2, C_{D}=\frac{24}{\mathrm{Re}}$;

- transient flow regime, $1<\operatorname{Re}<10^{3}: \mathrm{Nu}=2+0.6 \operatorname{Re}^{1 / 2} \operatorname{Pr}^{1 / 3}, C_{D}=\frac{24}{\operatorname{Re}}+\frac{4}{\sqrt[3]{\operatorname{Re}}}$

- turbulent flow regime, $\operatorname{Re}>10^{3}: C_{D}=0.44=$ const, $\mathrm{Nu}=\frac{0.37 \mathrm{Re}^{0.8} \operatorname{Pr}}{1+2.443 \operatorname{Re}^{-0.1}\left(\operatorname{Pr}^{2 / 3}-1\right)}$.

Critical value of the Bond is accepted as a criterion for droplet splitting due to Rayleigh-Taylor instability, due to Kelvin-Helmholtz instability - critical Weber number. To take into account the aerodynamic droplet splitting, the Weber and Bond criteria were calculated: Bo $=4 \rho_{s} \omega r_{s}^{2} / \sigma(\omega$ is the acceleration of the mass forces, $\sigma-$ is the surface tension) and We $=2 \rho\left|\vec{U}-\vec{U}_{s}\right|^{2} r_{s} / \sigma[6]$. It was assumed that on reaching the critical value of Bond number $\mathrm{Bo}_{*}=90$ or critical value of Weber number $\mathrm{We}_{*}=17$, the droplet splits into two spherical droplets of equal masses [6].

Mass evaporation rate due to the diffusion of fluid through the surface of the drop is determined by the formula [7]:

$$
G_{s}=4 \pi r_{s}^{2} k \frac{X}{1-X}
$$

where $k$ is the coefficient of the mass transfer; $X$ is mole fraction of the droplet matter vapor near its surface.

Having expressed $X$ in (2) through the vapor partial pressure $p_{0}$, we can write equation for a change of droplet radius in (1) in the following form:

$$
\frac{d r_{s}}{d t}=-\frac{k}{\rho_{s}} \frac{p_{0}}{p-p_{0}},
$$

where $p$ is the pressure of the ambient medium. 
The coefficient of mass transfer $k$ is calculated by the formula [7]:

$$
k=\frac{c_{f} D_{f} M_{f}}{2 r_{s}}\left[2+0.6\left(\frac{2 r_{s}\left|\vec{U}-\vec{U}_{s}\right| \rho_{s}}{\mu_{f}}\right)^{1 / 2} \cdot\left(\frac{\mu_{f}}{\rho_{f} D_{f}}\right)^{1 / 3}\right],
$$

where $D_{f}, c_{f}, \rho_{f}, M_{s}, \mu_{f}$ are the coefficient of binary diffusion, the summarized (air and kerosene vapors) volume mole concentration, the summarized density of air and kerosene vapor mixture, molecular mass of the drops substance and the dynamic air viscosity coefficient at the film temperature $T_{f}=\left(T_{f}+T\right) / 2$.

Diffusion coefficient in binary gas systems at low pressure was calculated by the Fullers-ShletterGiddings method [8]:

$$
D=\frac{T^{1.75}\left[\left(M_{A}+M_{B}\right) /\left(M_{A} M_{B}\right)\right]^{0.5}}{p\left[\left(\sum V_{A}\right)^{1 / 3}+\left(\sum V_{B}\right)^{1 / 3}\right]^{2}},
$$

where $M_{A}$ and $M_{B}$ are the molecular masses of $A$ and $B$ (liquid droplets and air); $\sum V_{A}, \sum V_{B}$ are the molecular diffusion volumes. The values of molecules diffusion volume for different substances are listed in [7]. It was obtained a formula for determining the diffusion coefficient drops of kerosene in the air on the basis of (5):

$$
D_{f}=\frac{C}{p}\left(\frac{T_{f}}{273}\right)^{1.75}
$$

where $C=1.22$ is for kerosene.

The efficient implicit difference scheme was used in the integration of (1). Using of the sustainable computational algorithm with variable step of integration allows us to control of the sharp change in the parameters of the environment during the process of calculation, in particular the temperature gradient and changing of the droplets parameters due to splitting.

Mathematical model (1) is supplemented for clarify of the initial droplet size in the calculation of their movement in the atmosphere in conditions of emergency with given that ejected fuel adjudged initially into the "footprint" - cocurrent flow of air entrained by the aircraft wing. The half-width of footprint $\delta$ behind the wing of the aircraft and the air velocity profile in the footprint were determined by the formulas $[9,10]$ :

$$
\delta \approx\left(\frac{F x}{\rho U_{f}^{2}}\right)^{1 / 3}, \quad \frac{u_{0}}{u_{f}}=\left(1-\eta^{3 / 2}\right)^{2},
$$

where $F$ is the ascensional wing power; $U_{f}$ is the incident flow velocity to the wing (velocity of the plane); $u_{0}$ is air velocity in the footprint in a fixed coordinate system; $\eta=y / \delta, y$ is the distance from the axis of footprint. Thus, when $\eta=0$, the velocity of air in the footprint is maximum. On the footprint border $(\eta=1)$, the velocity of air in the footprint is equal wind velocity.

The characteristic time $t_{*}$, through which the splitting drops occurs determined by the formula [11]:

$$
t_{*}=\frac{2 r_{s}}{\left|\vec{U}_{f}-\vec{U}_{s}\right|}\left(\frac{\rho_{s}}{\rho}\right)^{1 / 2} .
$$

Analysis of the results of a parametric study shown that regardless of the polydisperse initial composition the droplets of kerosene on the border of the footprint are the same size due to the numerous splitting in the footprint. Value of the minimum size of the droplets that adjudged out of the footprint to the "unperturbed atmosphere", depends only on the velocity of the plane. In particular, kerosene droplets 


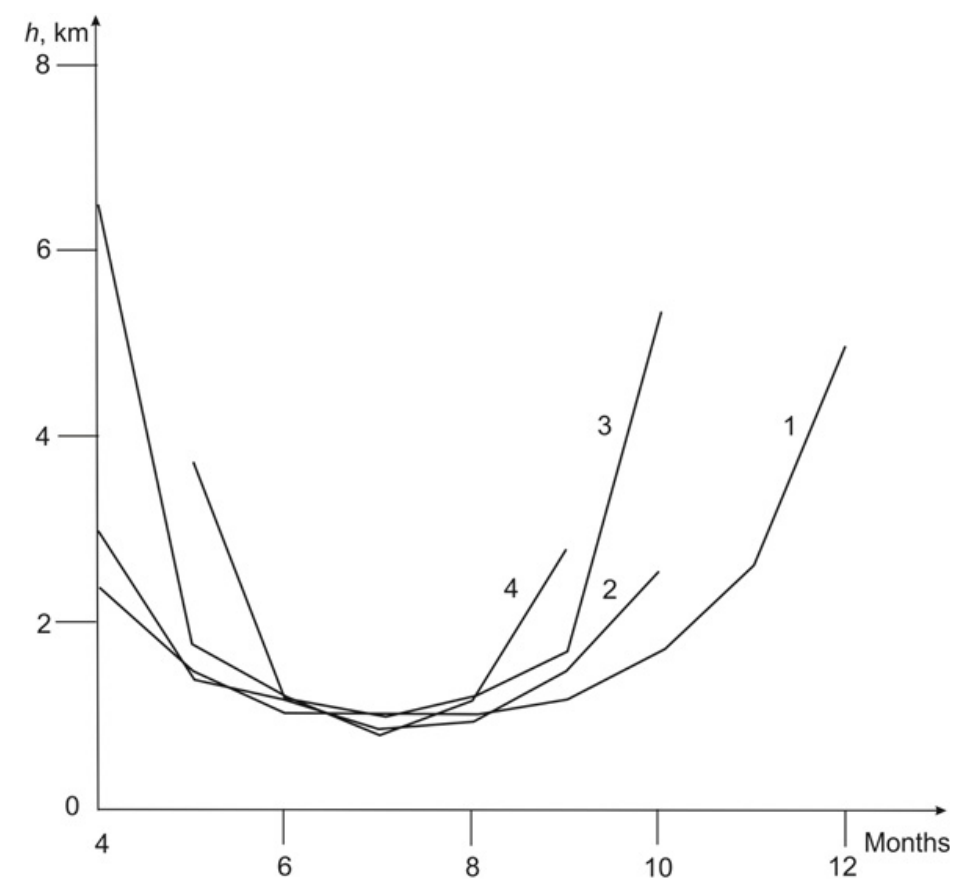

Figure 1. Heights of complete evaporation of the droplet. 1 - Kaliningrad region; 2 - Moscow region; 3 - Western Siberia; 4 - The Republic of Sakha (Yakutia).

radius is $\sim 0.5 \mathrm{~mm}$ when the velocity of aircraft of $600 \mathrm{~km} / \mathrm{h}$, and $\sim 0.4 \mathrm{~mm}$ when the velocity of aircraft of $900 \mathrm{~km} / \mathrm{h}$. In this connection the modeling was performed for monodisperse liquid-droplet cloud with variable initial drop size.

\section{Analysis of the results of numerical studies}

The optimal emergency discharge height of kerosene (height of complete evaporation of the droplet) for different Russian regions was evaluated based on the proposed mathematical model (1). There are data (Fig. 1) obtained by taking into account the meteorological conditions in the examined regions in different months of the year when the aircraft speed of $600 \mathrm{~km} / \mathrm{hr}$.

A mass of non-evaporated kerosene for different months for all regions when velocity of aircraft of $600 \mathrm{~km} / \mathrm{h}$ is shown in Table 1 . Averaged over the time distributions of atmospheric parameters were taken from [2].

Currently dumping of aviation fuel in emergency situations over the territory of Western Europe is governed by the rules of ICAO (International Civil Aviation Organization). Minimum (permissible) drop height kerosene $h_{\min }=1850 \mathrm{~m}$ during upward aircraft motion along longest spiral is set by ICAO rules. In this case it is allowed that $\sim 8 \%$ of the fuel not evaporate and reaches the earth's surface. Kaliningrad region of the Russian Federation is the closest under the season-climatic terms to Western Europe. Comparison of heights of the complete evaporation for the Kaliningrad region in different months at different aircraft velocities are shown in Fig. 2. It is found that ICAO rules are executed for the Kaliningrad region when a velocity of $900 \mathrm{~km} / \mathrm{h}$ (cruising aircraft velocity such as Boeing) in any months except January (16\%) and February (14.9\%).

The values of the precipitated on the surface mass of kerosene for four months, in which drop height exceed the permitted limit height $h_{\min }$ by the ICAO rules, are shown in Table 2 . 
Table 1. Mass of non-evaporated kerosene.

\begin{tabular}{|l|c|c|c|c|}
\hline \multirow{2}{*}{ Month } & \multicolumn{3}{|c|}{ Mass of kerosene which precipitated onto the earth's surface, \% } \\
\cline { 2 - 5 } & Kaliningrad region & Moscow region & Western Siberia & $\begin{array}{c}\text { The Republic of Sakha } \\
\text { (Yakutia) }\end{array}$ \\
\hline October & & & & 27 \\
\hline November & & 5 & 27.7 & 69.5 \\
\hline December & & 23 & 38.4 & 77.7 \\
\hline January & 9.7 & 37 & 49.1 & 77.2 \\
\hline February & 10.8 & 29 & 49.8 & 67.4 \\
\hline March & 2 & 22.7 & 26.1 & 38 \\
\hline April & & & & \\
\hline
\end{tabular}

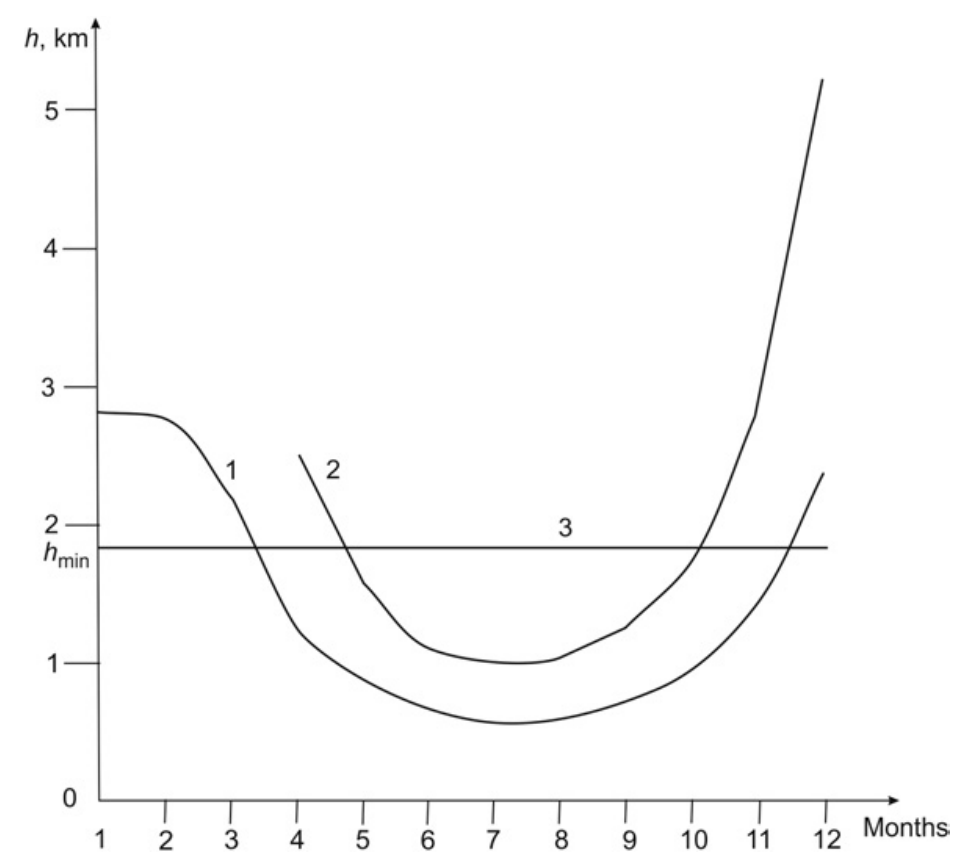

Figure 2. Heights of the complete evaporation for the Kaliningrad region. 1 - aircraft velocity is $900 \mathrm{~km} / \mathrm{h}$; 2 - aircraft velocity is $600 \mathrm{~km} / \mathrm{h} ; 3$ - allowable drop height (according to the ICAO rules).

Table 2. The precipitated on the surface mass of kerosene.

\begin{tabular}{|c|c|c|c|c|}
\hline Month & December & January & February & March \\
\hline The precipitated on the earth's surface mass of kerosene, \% & 8.1 & 16 & 14.9 & 3.2 \\
\hline
\end{tabular}

\section{Conclusion}

The results of numerical studies have shown the adequacy of this mathematical model to the physical process of the evolution of pollutants in the atmosphere in case of emergency or forced reset of kerosene from the aircraft. The developed model can be used to select the height limit of reset kerosene, guaranteeing complete evaporation of the droplets in the atmosphere to the different aircraft velocities. Opportunity to consider the impact of external factors associated with seasonal and climatic conditions and with peculiar of concrete region, for the choice of limit height of reset kerosene in emergency situation is an advantage of the model. This is especially important to fly over the territory of Russia. 


\section{References}

[1] V. Arkhipov, I. Zharova, Ye. Kozlov, A. Tkachenko, Atmospheric and Oceanic Optics, 26, 636 (2013)

[2] New aeroclimatic Directory free of atmosphere over the USSR (Moscow, Gidrometeoizdat, 1979)

[3] A. Adam, V. Arkhipov, V. Burkov, I. Plekhanov, A. Tkachenko, Atmospheric and Ocean Optics, 21, 437 (2008)

[4] G. Happel, T. Brenner, Hydrodynamics at low Reynolds numbers (Moscow, Mir, 1976)

[5] V. Levich, Physico - chemical hydrodynamics (Moscow, Gos. Izd. Phys.-math. lit., 1959)

[6] I. Vasenin, V. Arkhipov, V. Butov, A. Glazunov, V. Trofimov, Gas Dynamics of Binary Flows in Nozzles (Tomsk, TSU Publishing House, 1986)

[7] R. Berd, V. Stewart, and I. Lightfoot, Transport Phenomenon (Moscow, Chemistry, 1974)

[8] R. Rid, J. Prausnic, T. Sherwood, Properties of Liquids and Gases (Leningrad, Chemistry, 1982)

[9] G. Abramovich, Applied Gas Dynamics, (Moscow, Nauka, 1991)

[10] L. Landau, Ye. Liphshits, Theoretical Physics, 6 (Moscow, Nauka, 1986)

[11] R. Nigmatulin, Dynamics of Multiphase Media, 1 (Hemisphere, N.Y., 1990) 